\title{
Desarrollo de software para la gestión del mantenimiento en los laboratorios de la I.U. Pascual Bravo
}

\section{Software development for maintenance management in the laboratories of I. U. Pascual Bravo}

\author{
María Isabel Ardila-Marín ${ }^{a}$, William Orozco-Murillo ${ }^{b}$, Julián Galeano-Echeverri \& Andrés \\ Mauricio Medina-Escobar \\ a Docente, Facultad de Ingeniería, Institución Universitaria Pascual Bravo, Medellín, Colombia. \\ isabel.ardila@pascualbravo.edu.co \\ ${ }^{b}$ Docente, Facultad de Ingeniería, Institución Universitaria Pascual Bravo, Medellín, Colombia. \\ william.orozco@pascualbravo.edu.co \\ c Docente, Facultad de Ingeniería, Institución Universitaria Pascual Bravo, Medellín, Colombia. \\ julian.galeano@pascualbravo.edu.co \\ a Tecnólogo de apoyo en Desarrollo de Software, Institución Universitaria Pascual Bravo, Medellín, Colombia. \\ andres.medina@pascualbravo.edu.co
}

Recibido: mayo 21, 2018. Aceptado: junio 29, 2018.

\begin{abstract}
Resumen
La importancia de las acciones de mantenimiento en el sector de bienes y servicios para garantizar la disponibilidad y confiabilidad necesaria en los activos, ha generado la necesidad de tener herramientas que mejoren la administración de los departamentos de mantenimiento; es por esto que los Sistemas Computarizados para la Gestión del Mantenimiento (CMMS) aparecen para contribuir con la planeación, ejecución y control de las actividades en tales departamentos, haciendo que los datos obtenidos se conviertan en información relevante para la toma de decisiones para mejoramiento continuo. Para la I.U. Pascual Bravo, institución de educación superior con laboratorios para la prestación de servicios educativos y productivos, es fundamental trabajar en la conservación y seguridad de sus activos a través de la gestión del mantenimiento; por esto, se genera la necesidad de desarrollar un CMMS adaptado a las características de los activos de los diferentes laboratorios de la Facultad de Ingeniería, para centralizar la información de mantenimiento. En el presente artículo se revisa la evolución y desarrollo de los CMMS y se describe el diseño y la funcionalidad de SIMA, Sistema de Información de Mantenimiento, aplicación para centralizar la gestión del mantenimiento de los equipos de los laboratorios de la Facultad de Ingeniería. Con este módulo se pretende contribuir con la creación de cultura de mantenimiento, apoyando el correcto registro de la información para optimizar tanto las actividades y los recursos disponibles.
\end{abstract}

Palabras Clave: Gestión del mantenimiento, CMMS (Sistema Computarizado para la Gestión del Mantenimiento), indicadores de gestión, orden de trabajo, hoja de vida

\begin{abstract}
There is a growing importance of the maintenance actions in the productive sector, due to the requirement to guarantee the availability and reliability of the assets. This growing trend has generated the need for tools that improve the administration of the maintenance departments. In this context, Computerized Systems for Maintenance Management (CMMS) are in evolution, with the aim of contributing to the planning, execution, and control of the activities generated from maintenance operations. CMMS can make all the data collected from maintenance management tasks become relevant information for decision making in search of continuous improvement. I.U. Pascual Bravo is an educational institution with laboratories for the provision of both academic and industrial services. In these labs, it is essential to work on the conservation and safety of the assets through maintenance management activities. In consequence, there is a clear need of developing a CMMS adapted to the characteristics of the equipment from the different laboratories of the Faculty of Engineering, looking for the centralization of the operation and maintenance information. This article presents the evolution and development of the CMMS and then describes the functionality of SIMA. SIMA is an application developed to centralize the maintenance management of the equipment of the laboratories of the faculty of engineering. This software module intends to contribute to the creation of a maintenance culture supported by the correct recording of the generated information to improve both the completed activities and the available resources.
\end{abstract}

Keywords: Maintenance management, CMMS, Computerized System for Maintenance Management, management indicators, work order, datasheet.

\section{Citar como:}

M. Ardila-Marín, W. Orozco-Murillo, J. Galeano Echeverri, A. Medina-Escobar. "Desarrollo de software para la gestión del mantenimiento en los laboratorios de la I.U. Pascual Bravo". Revista CINTEX, Vol. 23(1), pp. 43-50. 2018. 
Desarrollo de software para la gestión del mantenimiento en los laboratorios de la I.U. Pascual Bravo Software development for manteinance management in the laboratories of I. U. Pascual Bravo

\section{INTRODUCCIÓN}

La gerencia del mantenimiento ha recibido una mayor atención por el aumento de la competencia mundial en la manufactura, ya que muchas compañías están buscando maneras de ganar competitividad sacando ventajas con respecto a los costos, el servicio, la calidad y la puntualidad en las entregas, y es en tales ventajas donde el papel que desempeña el mantenimiento es fundamental por su contribución a la productividad general de la organización [1].

Por esta razón se ha generado la necesidad de enlazar la gestión del mantenimiento con una herramienta informática que permita la planeación, ejecución y control de todas las acciones que se derivan de su misión como mantener funcionales y seguros todos los activos de una compañía; donde dichas acciones arrojan datos que deben ser transformados en información para generar beneficios para la productividad y competitividad de las empresas al facilitar la toma de decisiones respecto a esta área.

Existe una costumbre generalizada en el personal de mantenimiento de generar registros de las inspecciones e intervenciones de modo manual, lo que dificulta el acceso a la información para mejorar procesos, controlar acciones y recursos, entre otros [2]. En las últimas dos décadas, los cambios en el entorno de producción han hecho que la tarea de tomar decisiones sobre la asignación de los recursos de mantenimiento y programación de los trabajos de esta área sea más difícil dado que más variables y consecuencias deben ser consideradas y requieren una mayor capacidad de procesamiento de información [3].

Hoy en día, las industrias requieren procedimientos de gestión global integrados a sistemas de información con el fin de gestionar y utilizar la información de todas las áreas y, por lo tanto, asegurar un buen comportamiento del proceso. Estos requerimientos tienen por solución el desarrollo de sistemas de detección de fallas, sistemas de diagnóstico y sistemas de toma de decisiones [4]. Las Tecnologías de la Información y las Comunicaciones (TIC) podrían ser una herramienta importante para alcanzar la eficiencia y la eficacia en el mantenimiento, siempre que se apliquen de manera correcta y relevante [5], [6].

En este sentido, Sistemas Computarizados para la Gestión del Mantenimiento (CMMS) surgen como el complemento ideal para la gestión del mantenimiento. Los CMMS son comunes en las industrias de hoy, pueden llevar un gran número de ventajas que incluyen el aumento de la productividad, la reducción de costos y la utilización efectiva de los activos en cualquier fábrica. La lista de CMMS que están disponibles en el mercado ha crecido muy rápidamente en los últimos años. Una empresa debe buscar un sistema que se adapte a sus necesidades y objetivos específicos, las decisiones de selección de CMMS implican la consideración simultánea de múltiples criterios, incluyendo factores tangibles e intangibles; la priorización de estos factores puede ser un gran desafío y una tarea compleja [7], [8].

Este artículo está organizado así: en la sección 2 se presenta la importancia que los CMMS han adquirido desde la década de los 90, cuando se empezaron a visualizar los beneficios en su implementación y luego se describe la arquitectura de la aplicación SIMA en la sección 3, con sus principales funcionalidades como generación de órdenes de trabajo, construcción de la hoja de vida para cada equipo registrado en la aplicación e indicadores que facilitan el control de las acciones de mantenimiento para su mejoramiento continuo.

\section{IMPORTANCIA DE LOS CMMS}

Desde el año 1995, Streichfuss presentó un CMMS que cumplía con las exigencias especiales de los vehículos de transporte en la minería de carbón, aunque su funcionalidad se puede adaptar a otras aplicaciones industriales. El sistema apoyaba la planificación y control de mantenimiento con el fin de proporcionar una gestión de datos libre de contradicciones y redundancias, de manera que pudiera generar una estrategia de mantenimiento comparando el historial de mantenimiento del equipo, generando a la vez confianza en el diagnóstico basado en la hoja de vida de los activos [9].

En este mismo año ya se hablaba de gestión de mantenimiento en una industria manufacturera, esta vez basado en el software "AUTOCAD-12", el cual se complementaba con bases de datos "BASE IV", ambos se unían mediante lenguaje de consulta estructurada (SQL). El método para la ejecución de dicha gestión empezaba con la representación mediante "AUTOCAD-12" de los equipos en planta, los cuales estaban directamente ligados a una base de datos de mantenimiento que se organizaba en el programa "BASE IV".

La compañía Forasol-Foramer de Holanda decide utilizar un software para administración de mantenimiento (CMMS), después de no obtener mejoras al haber utilizado durante dos años un sistema manual de mantenimiento basado en tarjetas históricas individuales (T-CARD BOARD). Con el objetivo de eliminar el tiempo no productivo, implementaron un sistema informatizado de gestión de mantenimiento compuesto por módulos interconectados que incluye la gestión de órdenes de trabajo, la programación de mantenimiento preventivo, historial de mantenimiento realizado en el equipo, costos de mantenimiento, control de stock de repuestos y la presentación de informes. En 1998 presentó todos los resultados después de cuatro años de experiencia operativa con el CMMS seleccionado, afirmando que su uso condujo a un mejor sistema de mantenimiento, junto 
con un informe de trabajo mejorado que indica precisamente que se ha completado el trabajo de mantenimiento, proporcionando una disminución del tiempo no productivo [10, p. 11].

Para el 2001, Gabbar Hossarn, Kazuhiko, \& Yukiyasu, proponen la unión estratégica de los facilitadores de mantenimiento, apoyándose en estudio de riesgos y operabilidad (HAZOP), análisis de modos y efectos de falla (FMECA) y análisis de árbol de falla (FTA), todos utilizados para analizar y evaluar los tipos de fallo en forma cuantitativa; estos, combinados con las funciones de distribución de probabilidad de Monte Carlo y Weibull, se emplean para optimizar las tareas de mantenimiento. Además, se desarrollaron modelos de actividad y funcionales del proceso de RCM mejorado, y se propusieron modificaciones a los diferentes módulos del CMMS, MAXIMO $尺$ [11]. Y en 2003 se presentó un estudio de caso para mostrar la efectividad de la solución propuesta con base en CMMS-RCM en la optimización del mantenimiento de una planta que aplicaba los enfoques tradicionales [12].

En 2004, O'donoghue examinó la base de diversas estrategias de gestión de mantenimiento internacionalmente utilizadas. Estas estrategias ayudan a la función de mantenimiento y permiten optimizarla. Se presta especial atención a los CMMS y cómo esta estrategia particular se implementó con éxito en una empresa de fabricación textil de tamaño mediano irlandesa [13]. Además, desde este mismo año se reporta la importancia de la gestión del mantenimiento en instituciones educativas [14].

Al año 2007, Cerrada propuso un modelo de referencia para la gestión de fallas en los procesos industriales. Este modelo se basa en un marco genérico que utiliza sistemas multi-agente para sistemas de control distribuido; en este sentido, el problema de la gestión de fallas es visto como un proceso de control de realimentación y las acciones están relacionadas con la toma de decisiones: (a) en la planificación de tareas preventivas y (b) en la ejecución de tareas específicas de prevención y corrección. Un prototipo del sistema se desarrolla sobre el marco de desarrollo de Java® y un caso de estudio se presenta para la validación del prototipo [4].

En 2008, Kans desarrolló un modelo conceptual para identificar los requerimientos de $\mathrm{TI}$ en la gestión del mantenimiento, con su aplicación práctica en un proceso para la identificación de dichos requisitos. El proceso se ejemplifica en dos casos del mundo real. Por otra parte, este trabajo promueve el uso de un procedimiento estructurado para la identificación de las necesidades [5].

En 2014, Lin, mediante el uso de tecnologías de código de barras 2D y la identificación por radiofrecuencia (RFID - Radio Frequency Identification), presentó un novedoso sistema móvil basado en RFID para la gestión del mantenimiento [2], con mejoras en la gestión aplicada a equipos de laboratorio e instrumentos debido a que la gestión del mantenimiento eficaz puede ser extremadamente difícil dada la diversidad de equipos e instrumentos en un laboratorio que proporcionen una plataforma de intercambio de información de mantenimiento, puesto que están obligados a proporcionar un alto nivel de precisión y exactitud en sus pruebas. Para la integración de las tecnologías de información prometedoras, como los teléfonos inteligentes y agendas electrónicas habilitadas con RFID, el novedoso sistema propuesto se aplicó en un laboratorio de construcción en Taiwán y demostró las mejoras en el proceso de mantenimiento [2].

En el año 2015, la Universidad Agraria de La Habana (UNAH) reportó el desarrollo de una herramienta informática para apoyar el proceso de gestión de mantenimiento de equipos denominada SGMANTE [15], utilizando el lenguaje de programación Java; como entorno de desarrollo integrado (IDE) se utilizó MyEclipse Professional 2013, construido sobre la plataforma Eclipse que integra soluciones propietarias y de código abierto al entorno de desarrollo y como gestor de base de datos se utilizó Postgre SQL, este funciona muy bien con grandes cantidades de datos y una alta concurrencia de usuarios que acceden a la vez al sistema. Esta herramienta contó con cuatro módulos:

- Administración: abarca todo el control de usuarios del sistema, así como los privilegios para acceder a él.

- Patrimonio: controla todo el equipamiento del centro organizado según la estructura jerárquica de la universidad.

- Solicitud de servicio: es el encargado de gestionar todas las solicitudes de responsabilidades dentro del centro que hacen las distintas áreas a los especialistas de mantenimiento cuando se presenta un problema con algunos de sus equipos.

- Órdenes de trabajo: gestiona todas las órdenes que se abran a partir de una solicitud de mantenimiento.

En el mismo trabajo, se hacen recomendaciones sobre la importancia de definir cuidadosamente cada una de las actividades a informatizar, para definir los módulos necesarios y posteriormente integrarlos a una aplicación web y así elevar la eficiencia de la gestión del mantenimiento en la UNAH [15]. Adicionalmente, se debe considerar que el desarrollo de productos de software implica no solo escribir instrucciones de programación y ejecutarlas en un ordenador, sino que requiere cumplir los requisitos del usuario final, lo que implica establecer metodologías que ayuden a los profesionales a realizar su trabajo dentro de una serie de buenas prácticas en ingeniería del software para realizar una mejor planificación y seguimiento del trabajo [16]. 
Desarrollo de software para la gestión del mantenimiento en los laboratorios de la I.U. Pascual Bravo Software development for manteinance management in the laboratories of I. U. Pascual Bravo

En 2016, Nutdanai, Pornthip y Sanpanich [17] desarrollan una aplicación web informática para mejorar la gestión de equipos médicos en hospitales, con el objetivo de disminuir los costos de dicha gestión cuando es subcontratada y evitar cambio de bases de datos al cambiar de proveedor de la gestión de equipos, aumentando la seguridad en los datos durante la vida útil de los equipos. Se utilizó leguaje PHP y base de datos MySQL como herramientas de desarrollo y se probó en tres hospitales durante tres meses con resultados de evaluación satisfactorios. El programa incorpora seis módulos: registro de equipo médico, registro de repuestos, reparación y mantenimiento, mantenimiento preventivo y calibración, equipo médico en stock y resumen e informe [18].

\section{DESARROLLO DEL CMMS "SIMA"}

Dentro del contexto reportado en la sección anterior, se evidencia la importancia de los CMMS para apoyar las tareas de mantenimiento. Precisamente, la necesidad de centralizar la información de las acciones de mantenimiento en los laboratorios de la Institución Universitaria Pascual Bravo para facilitar su planeación y posterior control, sumada a los altos costos de las aplicaciones informáticas para administrar la gestión del mantenimiento que se encuentran en el medio, generó la necesidad de desarrollar al interior de la institución un CMMS para tal fin. El CMMS (Computerized Maintenance Management System - Sistema computarizado para la administración del mantenimiento) diseñado para la Institución Universitaria Pascual Bravo recibe el nombre de SIMA (Sistema de Información para Mantenimiento).

\subsection{Arquitectura de SIMA}

SIMA fue concebido bajo el patrón MVC - Model-View-Controller [19] el cual proporciona mayor nivel de abstracción y aumenta los niveles de seguridad sin contar con que, adicional a esta arquitectura, fue separada la capa de datos del Controlador y las consultas SQL en procedimientos almacenados (Stored Procedures) independientes que son accedidos por ejecución directa en el servidor de Base de Datos, con lo que se reducen totalmente los ataques por SQL-Injection.

La aplicación fue diseñada para que fuera de despliegue rápido, de fácil mantenimiento y de acceso desde diferentes plataformas y dispositivos. La base de datos relacional permite almacenar los datos más relevantes de los equipos al igual que la documentación, manuales, catálogos y demás información adicional a cada equipo.

La interfaz fue diseñada pensando en el diseño visual de Material Design [20], la cual posee diseños con colores sobrios y que permite el trabajo por tiempo prolongado en la aplicación con el fin de sacar el mayor provecho, incrementar su nivel de usabilidad y reducir los efectos visuales generados por cansancio o fatiga ocular, además de mantener un acceso rápido a la información gracias a su navegación simplificada, cumpliendo las normas ISO/IEC 9126 y ISO/IEC 25000.

\subsection{Generación de órdenes de trabajo}

El desarrollo del módulo SIMA busca centralizar los datos que arrojan las acciones de mantenimiento que se realizan a los equipos con los que cuenta la Institución Universitaria Pascual Bravo en los diferentes laboratorios de la Facultad de Ingeniería. Es fundamental generar a través de esta herramienta la cultura del registro para optimizar las acciones que buscan mantener disponibles y seguros los activos con los que la Institución cuenta para la formación de los estudiantes y la prestación de servicios al sector productivo.

El módulo está creado para generar Órdenes de Trabajo (OT) a partir de una programación de mantenimiento preventivo y, en la medida en que ejecuten las actividades y se registren los datos en SIMA, se conforma la hoja de vida para cada equipo. El menú principal del módulo de mantenimiento ofrece las siguientes funciones: programación mantenimiento, órdenes de trabajo, equipo, almacén, reportes, bitácora, leyes y normativas, administración.

El esquema para la programación de las órdenes de trabajo requiere inicialmente los registros de equipos, subsistemas y componentes, y verifica si cada rutina está en estado de cerrada o cancelada; con la holgura establecida, la frecuencia de dicha rutina y su duración; con esta información se genera una nueva orden de trabajo en estado "abierta" para que sea atendida por el equipo de mantenimiento (ver figura 1). 


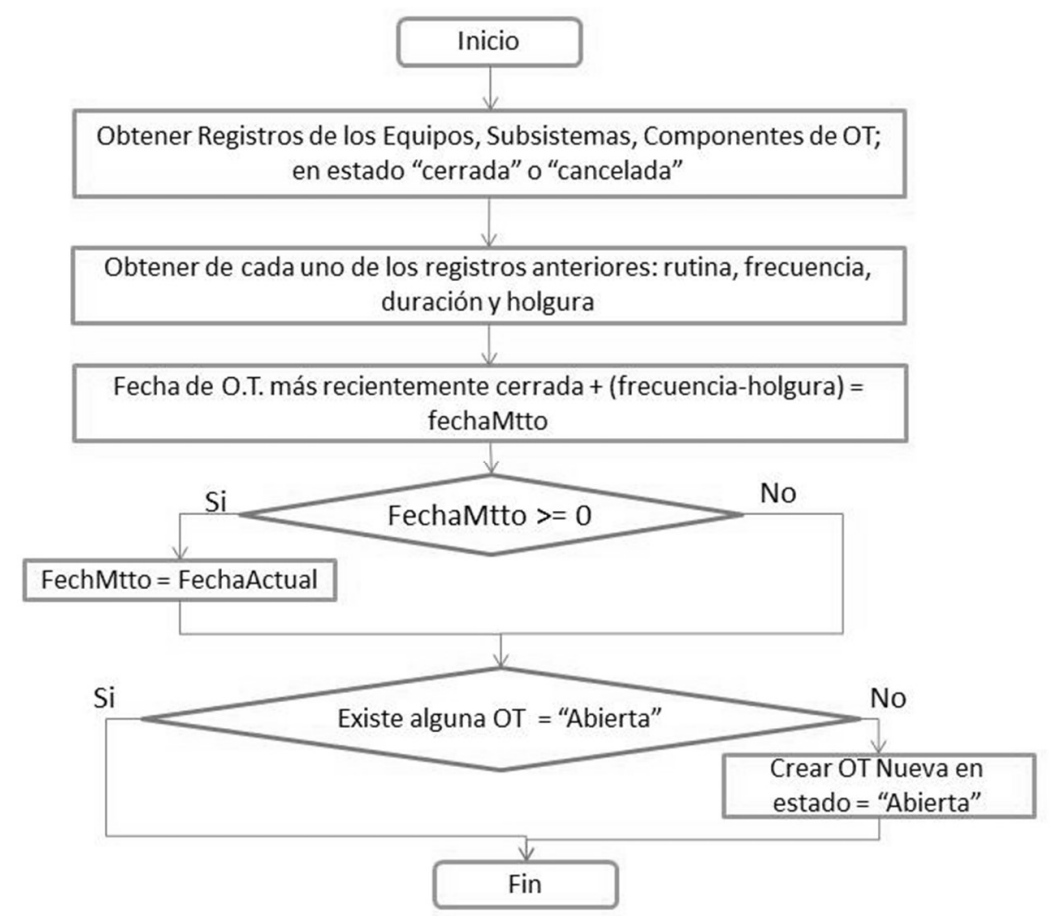

Figura 1. Esquema programación de órdenes de trabajo en CMMS SIMA

\subsection{Módulo "Equipo"}

En la opción equipo, como se ve en la figura 2, se guardan las especificaciones técnicas, los componentes, cartas de lubricación, programas de mantenimiento, órdenes de trabajo, catálogos y manuales y los protocolos de seguridad. De este modo, toda la información concerniente al equipo se centraliza para optimizar las acciones de mantenimiento y tener mayor control sobre su desempeño.
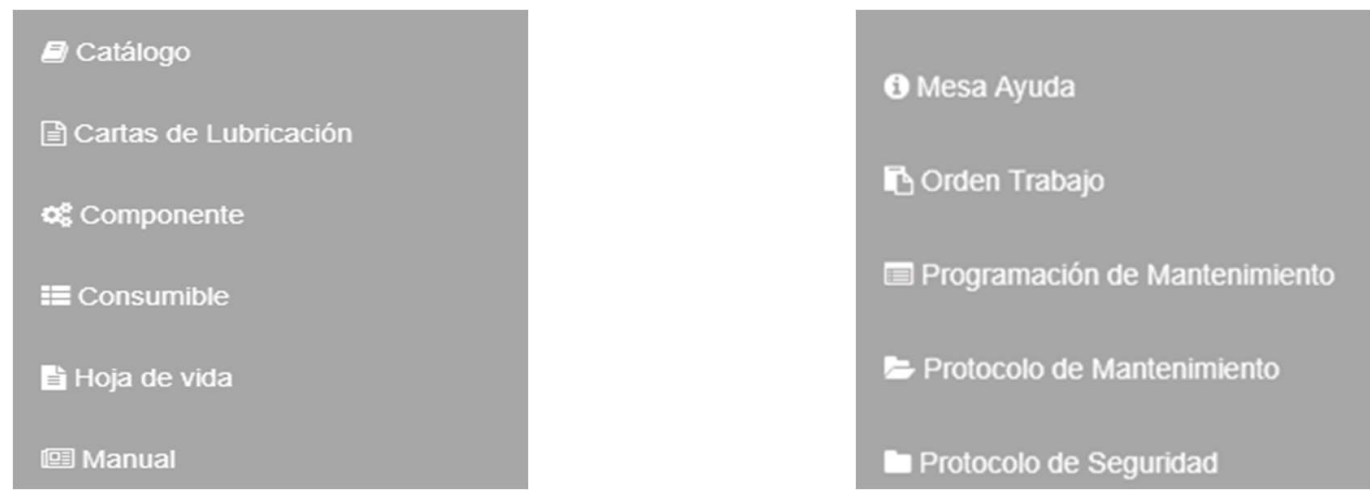

Figura 2. Opciones disponibles en el Menú desplegable "Equipo" de SIMA. Parte superior (izq.) y parte inferior (der.)

Además de encontrar la información mencionada para cada equipo, el módulo genera alertas con la holgura establecida en la programación del mantenimiento preventivo, de modo que se conozca con anterioridad la intervención que debe hacerse para realizar la gestión concerniente a los recursos necesarios para su ejecución.

Cabe resaltar que el módulo genera automáticamente las órdenes de trabajo (de acuerdo con la programación de mantenimiento) a partir de que se han cerrado estas después de cumplida la intervención y de haberse alimentado inmediatamente la hoja de vida de cada equipo (como lo ilustra la Figura 3), lo que genera un archivo histórico de intervenciones, elemento fundamental para controlar y optimizar el programa de mantenimiento. La información que contiene la orden de trabajo y por tanto la hoja de vida es: equipo, componente, fecha, descripción de la actividad, prioridad de la intervención. Para cerrar esta y alimentar la hoja 
de vida se debe ingresar la siguiente información: fecha de intervención, tipo de mantenimiento, descripción general, horas de intervención, repuestos utilizados, responsable de la actividad y observaciones.

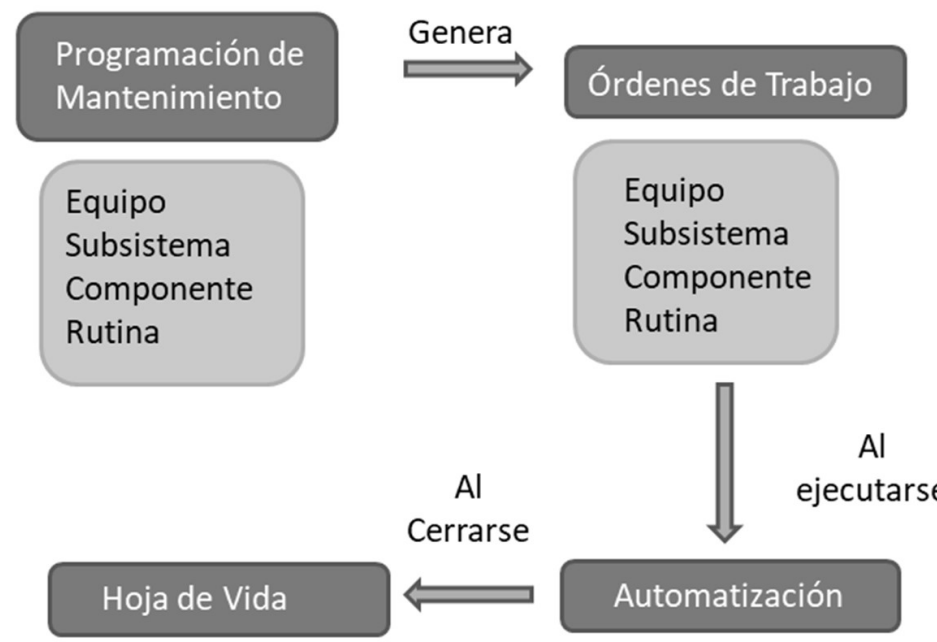

Figura 3. Secuencia de generación de Órdenes de Trabajo y Hoja de vida en el CMMS SIMA

La aplicación web utiliza como motor de base de datos MySQL, en el que las consultas son accedidas a través de Stored Procedures (SP) y ejecutadas por el servidor de Base de Datos para incrementar su seguridad al retirar el script de SQL de la capa de modelo, pasando solo parámetros de ejecución para el SP. La capa visual fue construida con HTML5, CS3, JavaScript, PHP articulado con Bootstrap, lo que permite que sea posible desplegar en diferentes dispositivos con conexión a internet (PC y móviles: tablets y celulares), multi-dispositivo y multiplataforma, gracias a la arquitectura Modelo-Vista-Controlador (MVC), y con un mejor modelo de seguridad con los respectivos roles y validación de permisos (Figura 4). El patrón MVC usado se creó directamente con PHP, para mejorar el control sobre el proceso de comunicación en el patrón en cada uno de los módulos de la aplicación [21].

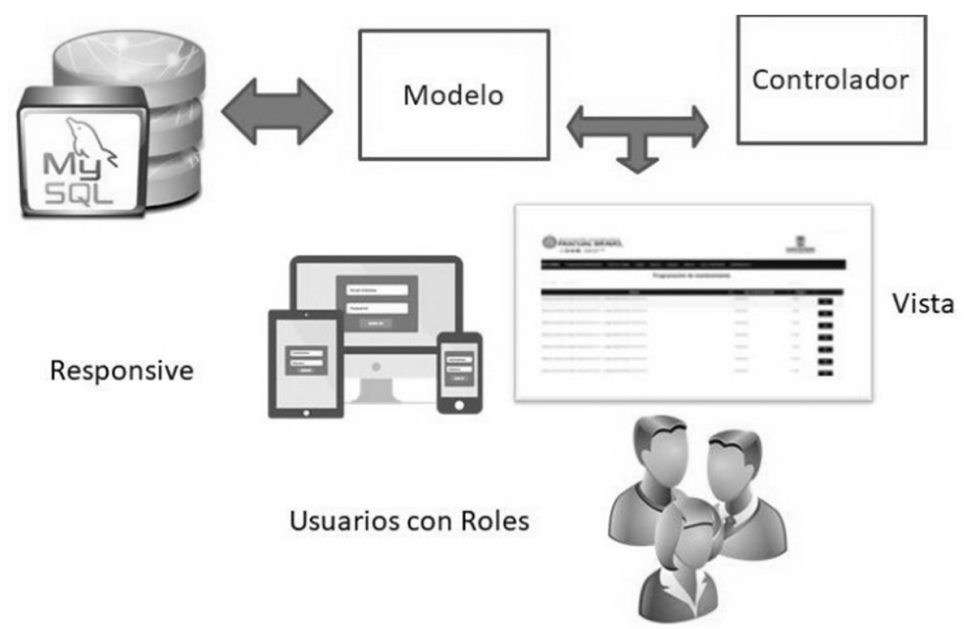

Figura 4. Diagrama de procesos para la aplicación Web para el CMMS SIMA

\subsection{Indicadores de proceso a generar}

El módulo genera unos reportes iniciales respecto al cumplimiento de lo programado; es decir, arroja indicadores sobre órdenes de trabajo cerradas vs las planeadas, cantidad de órdenes de mantenimiento preventivo vs órdenes totales, cantidad de órdenes de mantenimiento correctivo vs las totales y número de intervenciones realizadas por personal propio vs las realizadas por personal subcontratado. Con estos indicadores se espera conocer la gestión del área de mantenimiento para pasar a su optimización.

Luego de culminada la etapa de diseño se realizará una prueba piloto en un laboratorio del programa de Mecánica, otro del programa de Eléctrica y otro del programa de Electrónica para verificar los beneficios que 
genera centralizar la información en la toma de decisiones a partir de los reportes del SIMA con los datos que recopila de las diferentes acciones que se ejecutan en los equipos de los laboratorios y conservar su función.

\section{CONCLUSIONES}

Los CMMS además de ser fundamentales en la gestión del mantenimiento son una herramienta para crear la cultura del registro que tanto requieren las empresas para optimizar sus procesos y el uso de los recursos con los que cuenta.

El desarrollo del módulo SIMA obedeció a la necesidad de centralizar la información que arrojan las actividades de mantenimiento de los equipos que hacen parte de los diferentes laboratorios de la Facultad de Ingeniería de la I.U. Pascual Bravo. La generación automática de las órdenes de mantenimiento de acuerdo con los programas preestablecidos en el módulo pretende facilitar la ejecución de las actividades y el registro de los detalles de estas luego de cumplida cada intervención para beneficiar el control de todo el plan, el personal y los recursos por utilizar.

\section{AGRADECIMIENTOS}

Los autores agradecen el apoyo de la Institución Universitaria Pascual Bravo mediante al proyecto No. IN201608 para el desarrollo del software SIMA.

\section{REFERENCIAS}

[1] M. Faccio, A. Persona, F. Sgarbossa, and G. Zanin, "Industrial maintenance policy development: A quantitative framework," Int. J. Prod. Econ., vol. 147, pp. 85-93, Jan. 2014.

[2] Y.-C. Lin, W.-F. Cheung, and F.-C. Siao, "Developing mobile 2D barcode/RFID-based maintenance management system," Autom. Constr., vol. 37, pp. 110-121, Jan. 2014.

[3] L. Swanson, "An information-processing model of maintenance management," Int. J. Prod. Econ., vol. 83, no. 1, pp. 45-64, Jan. 2003.

[4] M. Cerrada, J. Cardillo, J. Aguilar, and R. Faneite, "AGENT-BASED MAINTENANCE MANAGEMENT

SYSTEM FOR THE DISTRIBUTED FAULT TOLERANCE,” IFAC Proc. Vol., vol. 39, no. 13, pp. 938-943, 2006.

[5] M. Kans, "An approach for determining the requirements of computerised maintenance management systems," Comput. Ind., vol. 59, no. 1, pp. 32-40, Jan. 2008.

[6] J. J. Castro Maldonado, J. A. Patiño Murillo, and C. Gómez López, "Procesos de I+D+i en el Centro de Servicios y Gestión Empresarial del Servicio Nacional de Aprendizaje SENA 2015-2017," Rev. Espac., vol. 39, no. 20 , p. $21,2018$.

[7] O. Durán, "Computer-aided maintenance management systems selection based on a fuzzy AHP approach," Adv. Eng. Softw., vol. 42, no. 10, pp. 821-829, Oct. 2011.

[8] L. F. Jaramillo et al., "Modificación y Evaluación de un Lubricante con Nanotubos de Carbono para Aplicaciones en Sistemas Rodantes-Deslizantes," Rev. CINTEX, vol. 20, no. 2, pp. 9-33, 2015.

[9] M. Streichfuss and P. Burgwinkel, "An expert-system-based machine monitoring and maintenance management system," Control Eng. Pract., vol. 3, no. 7, pp. 1023-1027, Jul. 1995.

[10] R. Moore, Ed., "Chapter 11 - Implementing a Computerized Maintenance Management System," in Making Common Sense Common Practice (Third Edition), Third Edition., Boston: Butterworth-Heinemann, 2004, pp. 277288.

[11] H. A.Gabbar, K. Suzuki, and Y. Shimada, "Design considerations of computer-aided RCM-based plant maintenance management system," in Computer Aided Chemical Engineering, vol. 9, Elsevier, 2001, pp. 859864.

[12] H. A. Gabbar, H. Yamashita, K. Suzuki, and Y. Shimada, "Computer-aided RCM-based plant maintenance management system," Robot. Comput.-Integr. Manuf., vol. 19, no. 5, pp. 449-458, Oct. 2003.

[13] C. D. O'Donoghue and J. G. Prendergast, "Implementation and benefits of introducing a computerised maintenance management system into a textile manufacturing company," J. Mater. Process. Technol., vol. 153154, pp. 226-232, nov. 2004.

[14] W. Orozco Murillo, "Estructura conceptual de la línea de investigación en gestión del mantenimiento en el I.T.P.B.," Rev. CINTEX, vol. 10, pp. 64-69, 2004.

[15] Y. Suárez, D. Medina, and P. M. Hernández, "Sistema automatizado para la gestión del mantenimiento de equipos (módulos administración y solicitud de servicio)," Rev. Cienc. Téc. Agropecu., vol. 24, pp. 85-90, 2015. 
[16] A. Peralta and F. P. Romero, "Toma de Decisiones a partir de Conocimiento Extraído tras el Análisis de Comportamientos Previos. Aplicación Práctica para la Dirección de Proyectos de Desarrollo de Software," Rev. CINTEX, vol. 20, no. 2, pp. 97-111, 2015.

[17] S. Nutdanai, L. Pornthip, and A. Sanpanich, "Development of an information system for medical equipment management in hospitals," in 2016 9th Biomedical Engineering International Conference (BMEiCON), Laung Prabang, Laos, 2016, pp. 1-5.

[18] K. Fraser, H.-H. Hvolby, and T.-L. (Bill) Tseng, "Maintenance management models: a study of the published literature to identify empirical evidence: A greater practical focus is needed," Int. J. Qual. Reliab. Manag., vol. 32, no. 6, pp. 635-664, 2015.

[19] M. Aniche, G. Bavota, C. Treude, M. A. Gerosa, and A. van Deursen, "Code smells for Model-ViewController architectures," Empir. Softw. Eng., vol. 23, no. 4, pp. 2121-2157, Aug. 2018.

[20] N. Yu and J. Kong, "User experience with web browsing on small screens: Experimental investigations of mobile-page interface design and homepage design for news websites," Inf. Sci., vol. 330, pp. 427-443, Feb. 2016.

[21] R. E. Johnson, "Frameworks = (components + patterns)," Commun. ACM, vol. 40, no. 10, pp. 39-42, Oct. 1997. 